\title{
Using Information Retrieval Activities to Foster Analytical Thinking Skills in Higher Education in Thailand: A Case Study of Local Wisdom Education
}

\section{Puchit Puchumni ${ }^{1 \star(D)}$ \\ Sumalee Tungpradabkul ${ }^{2}$ Ratana Magee M $^{3}$}

'Department of Mathematics, Faculty of Education, Kanchanaburi Rajabhat University, Thailand Email:superchedi@hotmail.com Tel:+66860301269

${ }^{2}$ Department of Biochemistry, Faculty of Science, Mahidol University, Thailand

Email:pexcotung@gmail.com Tel: +66814393280

${ }^{3}$ Learning Promotion Counselor, Singapore

Email:mageeratana@gmail.com Tel:+6587258731

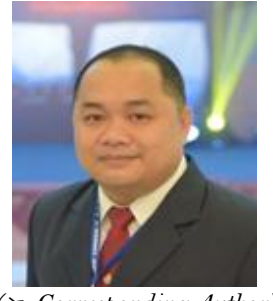

( Corresponding Author)

\section{Abstract}

Three information retrieving lessons were designed to foster analytical thinking in freshmen with minimal prior active learning experience. Thailand Local Wisdom contents were used as an online information platform due to the scarcity in well-established credible information sources. The lessons were collaborative learning both within own group and between groups. The information retrieving, credibility sorting, classroom sharing, analytical thinking assessments, and selfevaluating activities were conducted in sequential steps and repeated in three trials. A reflective pause was introduced between trials. The self-evaluating pause procedure using teacher's feedback was possibly a major reason for gains, from $65.0 \%$ to $82.5 \%$ and to $92.5 \%$, in the number of students exhibiting analytical thinking evidence. A free-response survey after the learning experience showed that the students express high appreciation for the active learning activities as fun and analytical-thinking-promoting learning experiences.

Keywords: Active learning, Local wisdom, Local knowledge, Information retrieval, Information credibility, Analytical thinking

Citation | Puchit Puchumni; Sumalee Tungpradabkul; Ratana Magee (2019). Using Information Retrieval Activities to Foster Analytical Thinking Skills in Higher Education in Thailand: A Case Study of Local Wisdom Education. Asian Journal of Education and Training, 5(1): 80-85.

History:

Received: 17 October 2018

Revised: 19 November 2018

Accepted: 31 December 2018

Published: 21 January 2019

Licensed: This work is licensed under a Creative Commons

Attribution 3.0 License $(\mathrm{cc})$ E

Publisher: Asian Online Journal Publishing Group
Contribution/Acknowledgement: All authors contributed to the conception and design of the study.

Funding: The authors wish to express their gratitude to the Office of the Higher Education Commission, Ministry of Education, Thailand for the financial support. Competing Interests: The authors declare that they have no conflict of

Transparency: The authors confirm that the manuscript is an honest, accurate, and transparent account of the study was reported; that no vital features of the study have been omitted; and that any discrepancies from the study as planned have been explained.

Ethical: This study follows all ethical practices during writing.

\section{Contents}

1. Introduction

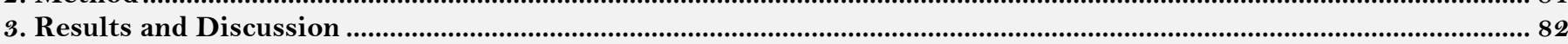

4. Conclusion 84

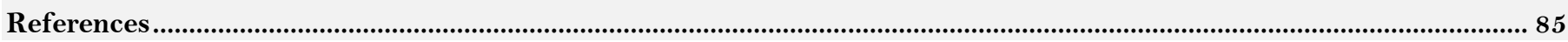




\section{Introduction}

Although active learning has been regularly practiced in schools in the US for decades and in many different forms, one of which is the opportunity for students to engage in retrieving, analyzing, and making use of obtained information vastly available on the Internet, only a tiny portion of non-science classrooms in Asia have witnessed such active learning activities, especially in Thailand education. While science classrooms can offer ample opportunities for students to be active in their learning, due to limited resources and the apparent inequality in education, Thai students in the rural areas do not necessarily have access to active science learning. Furthermore, non-science subjects, e.g. languages and social studies, often are conducted in a passive learning fashion. As a result, most rural students lack the opportunity to actively engage themselves in the higher-order thinking levels of learning; namely, analyzing, synthesizing and evaluating learning (Forehand, 2005).

Active learning was first introduced in Thailand's basic educational curriculum in 1999 and discussion of active learning classroom implementation at the national level started in early 2000 (Office of the Education Council, 1999). However, there have not been official mandates from the government for active learning implementation in any of the three types of Thai educational institutes : formal, non-formal, and informal. Attempts and initiatives to convert traditionally passive learning classrooms in some rural areas into active ones have been reported (Climer et al., 2009). Teemuangsai and Meesook (2017) reported that the classroom practices in 90 secondary schools (2430 students in Grades 7-12) in the Northeastern region of Thailand were traditional methods. The students in the study suggested a need for change in the teaching style to make the lessons less boring, more engaging and relevant to real life. In this study, we are presenting another example of a rare active learning practice in a Thai classroom aiming to foster analytical thinking skills through retrieving information activities on Thai Local Knowledge, also widely known as Local Wisdom. Several meanings of Local Wisdom are shown here.

Meaning 1: Local Wisdom (noun) - the knowledge that has been discovered or acquired by local people through long experience

The Bangkok Post

Meaning 2: Local Wisdom - A set of ideas or policies based on the values of the virtues found in a community and often applied, believed two be the guidance of life, and handed down from time to time (Sopa, 2018).

Meaning 3: Local Wisdom - Basic knowledge gained from living in balanced with nature and related to culture in the community which is accumulated and passed on (Mungmachon, 2012).

Thailand has enjoyed a reputation of richness in deeply rooted culture throughout its long history. It is the only nation in Southeast Asia that has never been governed by any western nations during the colonial period. As a result, indigenous people in different parts of Siam (former name of Thailand during the colonial days) had liberty in creating own ways of life without much of foreign influences. Through generations, these independent creations have become Indigenous Knowledge or Local knowledge or Local Wisdom. Over the years, many Thai governments put emphasis on the importance of the diversity in Local Wisdom but not until 1997 a Local Wisdom study and appreciation mandate at all educational levels was embarked by its inclusion in the Constitution of the Kingdom of Thailand B.E. 2540 (Office of the National Education Commission, 1997). Educational institutions in the provincial areas are strongly encouraged to integrate Local Knowledge contents in own areas into their lessons. The so called 'local curricula', embedding Local Knowledge contents, were built to offer learners to learn from real world and local communities (Henschke and Ratana-Ubol, 2015). Ratana-Ubol (2016) also pointed out the use of Local Wisdom as a lifelong learning tool in Thailand educational systems. As most of the Local Knowledge evolved many generations ago, by local people's ways of life, the original records of the associated knowledge and know-how are often non-existing. The acclaimed Local Knowledge contents have been verbally passed on from one generation of local folks to the next. It can be stated that the majority of the available Local Wisdom contents, in prints or online, are compilation work of the verbally transferred knowledge and know-how; thus, Thailand's Local Wisdom information seekers are presented with a challenge of reliability of the collected contents.

Kanchanaburi Rajabhat University, KRU, is one of the forty pioneer Rajabhat Universities whose main mission is to provide higher education, with the national mandate on Local Wisdom education and appreciation, to Thai students in the provincial areas. KRU is situated in the central-west region of Thailand in Kanchanaburi province which shares a rich culture with Myanmarese inhabitants along the borders between Thailand and Myanmar. The Local Knowledge education at KRU spreads throughout the four-year curriculum with a variety of learning activities: research, experiential learning through field studies, interviews and surveys, immersion in local communities, etc. Freshmen are usually tasked with an overview study of Kanchanaburi's local wisdom topics.

In this study, we created an opportunity for a group of freshmen to develop self-learning and analytical thinking skills by taking advantage of the lack of the original or official records of the Local Knowledge contents in Kanchanaburi. In three consecutive steps, a freshman student, working collaboratively with a peer and cross peer groups, evaluated retrieved information on Local Wisdom topic of choice from no fewer than two different online sources. The analyzed sets of information that appeared to be consistent or in-line among the searched sources were then deemed credible for future use. A free-response survey after the learning experience shows promising results in students' active learning appreciation and a gain in analytical thinking skills. Many other desirable byproducts, e.g. responsibility, self confidence in learning and decision making skills were also reflected in the students' self-reflection.

\section{Method}

The subjects of this study are a freshman group of 40 students who started their college study in 2011 in the Department of Mathematics, Faculty of Education, Kanchanaburi Rajabhat University (KRU) in Kanchanaburi province of Thailand. The subjects comprise of 11 male and 29 female rural students from Kanchanaburi province and its nearby provinces. They could be characterized as academically disadvantaged and largely ranked in the bottom half when compared with their peers in the central regions of Thailand. The subjects had no to little 
academic information retrieval skills including experience in evaluating retrieved information from the Internet. A list of Local Wisdom topics which are indigenous to Kanchanaburi province was provided by the teacher and the subjects, worked in groups of two, chose their topic of interest. In three consecutive steps, the students searched online information on three important aspects of the chosen topic: Aspect1 - origin, Aspect 2 - production methods and Aspect 3 - usage and applications. In each step, after an aspect's information search, group members reviewed, discussed, and compared and contrasted the obtained information. Groups with the same Local Wisdom topic performed a secondary cross-content analysis between groups. Each group was asked to reflect and share their learning experiences from the previous step in a whole classroom setting before starting the next aspect task. After each aspect tasks, the student independently submitted a report detailing personal work on three activities (Activity 1 - collected information substantiated by at least two sources, Activity 2 - bibliography details of the sources and Activity 3 - student's own analysis and evaluation details aiming at extracting credible information) to the teacher for assessments on punctuality, completeness and analytical thinking evidence. At the end of the retrieval, analysis and evaluation processes of all three aspects of the chosen Local Wisdom topic, the student was asked to independently perform a self-reflection evaluation on direct experience gained from these active learning lessons by answering free-response questions. The probing questions were

1. What is your general impression of these active learning lessons?

2. What skills do you think you have acquired during these active learning lessons?

\section{Results and Discussion}

There is a wide consensus among educators and administrators that the $21^{\text {st }}$ Century has provided students with flux of information over the Internet and on many different media platforms such as blogs, wikis, Facebook and Social Q\&A sites. Such information can come from many different types of sources, ranging from governmental agencies, well-known news organizations, self-made gurus, frequent bloggers to a lay person; thus, there is a need to validate the credibility of the obtained information (Kim, 2010). The active learning lessons in this study were designed to foster analytical thinking skills on information retrieval in an environment where reliable references are scarce and a multitude of online search results are readily available at the fingertip of the students. The information in the retrieving activities in this study was solely indigenous to Kanchanaburi habitats. The online media platforms employed in the search process comprised of multiple formats namely wikis, blogs and webpages. While there are many other information seeking goals when people perform an information search process, the most succinct or the most entertaining information rather than the most credible information possible as reported by Metzger et al. (2010) work, or the efficiency of the search process itself can be at the center of an information retrieval study, for example how to formulate effective keywords or which search engines would yield desirable results, the primary objective of our study focuses on students filter out credible information from Thai Local Wisdom online information by analyzing and evaluating the obtained information. In the traditional sense, information credibility are believed to be directly related to source credibility. As mentioned earlier in this report, there are no official collections of Thai Local Wisdom materials and the available Local Wisdom contents are merely compilation works of the verbally transferred knowledge from one generation to the next. These sets of knowledge are made available online by authos who may not necessarily have training and/or education relevant to Thai Local Knowledge. Metzger and Flanagin (2013) stated that credible sources of information are easily recognized by virtue of their observable and verifiable credentials which are rooted in specific qualifications or training. The specific qualifications or training in the field of Local Wisdom of most Thai Local Wisdom online sources were not readily observable or verifiable. As a result, we assume that the online sources are not necessarily credible sources on Thai Local Wisdom domain and the credibility of the searched results should be focused on the information not the sources. With this assumption in mind, we designed the active learning activities for the freshmen to evaluate the credibility of the information. We aimed that the students would gain some analytical thinking skills from repetitive analysis and evaluation steps. Content understanding and Local Wisdom appreciation aspets were also parts of the learning goals but will not be discussed in this report.

The subjects in this study were freshman starting their first semester at KRU in 2011. Since the college placement in Thailand was mainly based on the national admission system of academic achievements in high school years and the college admission test scores, it is safe to say that the academic standing of this group of students was in the lower half of their national peers. Their learning experiences in the primary and secondary school years prior to KRU admission can be described as traditionally passive, teachers passed on knowledge to students, which was also discussed in Teemuangsai and Meesook (2017) report. The students also had very little experience in actively acquiring information prior to participating in this study. It is also worth noted that they came from socioeconomically disadvantaged background when compared to their counterparts in the central regions of Thailand. KRU has launched a general education curriculum that requires all freshmen to earn three credit hours in Thai Living which encompasses National and Kanchanaburi Local Wisdom studies. The active learning lessons in this study were conducted in part with the Thai Living curriculum. The lack of active and analytical thinking learning experiences in their previous academic settings made these freshmen, future classroom teachers, suitable for this study where the authors integrated information retrieving activities and widely unevaluated online Local Wisdom contents into a learning means to promote the students' analytical thinking skills.

Each student worked collaboratively with a learning partner on their chosen topic of interest from a list of six Local Wisdom topics that are indigenous to the Kanchanaburi areas. The topics were 1. 100-Color Fabrics 2. Sangkra Fabrics 3. Gems 4. Pocket Knives 5. Bamboo Rice Mills and 6. Carved Wood Furniture. Each group conducted online information search from no fewer than two different sources on three aspects of the chosen Local Wisdom topic, namely, origin, production, and application. Each aspect task can be described in three activities: Activity 1 - information retrieval from multiple sources, Activity 2 - bibliography details and Activity-3 content analyses. A first analytical thinking opportunity arose when the group partners reviewed and analyzed the retrieved information. They compared and contrasted did the contents to filter out credible information using consistency heuristic which was reported in Metzger et al. (2010) work as a relatively fast and frugal means of arriving at the credible judgment of information. A second analytical thinking opportunity occurred when each 
group performed a cross-content analysis with other groups working on the same topic. At this cross-group checking, some groups were able to arrive at more consistent contents between groups which allowed the students to realize the importance of collaborative work that enabled them to gain more breadth in their search. In conclusion, after working on the three aspects of the local wisdom search, each student had six sessions to learn and practice analytical thinking skills. After each aspect, a classroom discussion followed in which the students shared their findings and simultaneously learned other Local Wisdom topics from their peers. Formative assessments on each student's report provided feedback on the previous aspect's performance prior to the start of the next one. Each student's evidence of analytical thinking was drawn from the three components of the submitted report: 1. data collection : from at least two sources, 2. analysis and evaluation : break down complex information to relavant smaller and manageable chunks and scrutinize the data for consistency, and 3. decision making : conclude credible information. Figure 1 shows a gain in the number of students who demonstrated evidence of analytical thinking. A possible explanation for the constant gains from Aspect $1(65.0 \%, \mathrm{n}=26)$ to Aspect $3(92.5 \%, \mathrm{n}=37)$ could be attributed to the strategic pause for self-review in between aspects that allowed some students to have more time to gain deeper understanding of the thinking process either by discussing with their peers or utilizing the teacher's feedback or both. Pause procedure has been reported to enhance student engagement and learning in various fields of study such as in medicines (Bachhel and Thaman, 2014) and in accounting (Chowdhury, 2016). The proposed reason for analytical thinking gains in this study is well in line with the effectiveness of pause procedure concluded in Bachhel et al. and Chowdhury's studies.

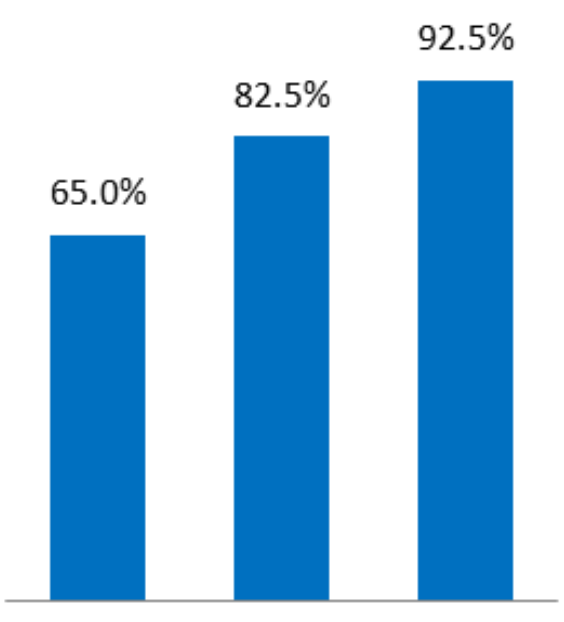

Aspect 1 Aspect 2 Aspect 3

Figure-1. Percent analytical thinking evidence after each aspect's activities

A further look at individual student's analytical performance in all three trials revealed that, when compared to the total number of students, both the $17.5 \%(\mathrm{n}=7)$ gain from Aspect 1 to Aspect 2 and 10.0\% $(\mathrm{n}=4)$ gain from Aspect 2 to Aspect 3 represented new students from the previous step. It can be concluded that $65.0 \%(\mathrm{n}=26)$ of the stubject group in this study consistently learned and practiced their analytical thinking skills in sorting credible information throughout the lessons. A 27.5 percentage $(n=11)$ of the students successfully demonstrated analytical thinking evidence after the first or second aspect tasks. At any stage of the three aspect search tasks, a 7.5 percentage $(\mathrm{n}=3)$ of the group did not exhibit minimum analytical thinking evidence in their content credibility discussions and reports. Lack of motivation and being extremely reluctant learners in nature could be plausible causes for the observed low performance. However, the exact cause of this learning behavior requires a further exploration.

Another analytical thinking evidence stemmed from the students' self reflection after the completion of the lessons. The students' answers to the open-ended questions -1 . What is your general impression of these active learning lessons? and 2. What skills do you think you have acquired during these active learning lessons? - shed some light on students' own thinking skill assessments. While other skills such as thoroughness, planning, observation, decision making and responsibility were reported in the students' answers (Figure 2), the thinking skill was widly cited by the students. $95.0 \%(\mathrm{n}=38)$ of the students indicated some versions of analytical thinking engagement in their responses. Examples of students' related responses are:

Student 1 By comparing materials, it helped me think and remember the contents better.

Student 19 This activity made me think and see more vieropoints.

Student 29 I will be more prudent (analytical thinking) in my future search.

Student 34. I liked Activity 3. I got to evaluate!

Student 35 I had to think analytically and really hard in this activity.

Some students $(30.0 \%, n=12)$ cited the specific 'analytical thinking' term in their free responses as an acquired skill after completing the lessons. Examples of students' related responses are:

Student 3 When we applied analytical thinking, I enjoyed this step!

Student 8 I know how to use analytical thinking to sort reliable information. 


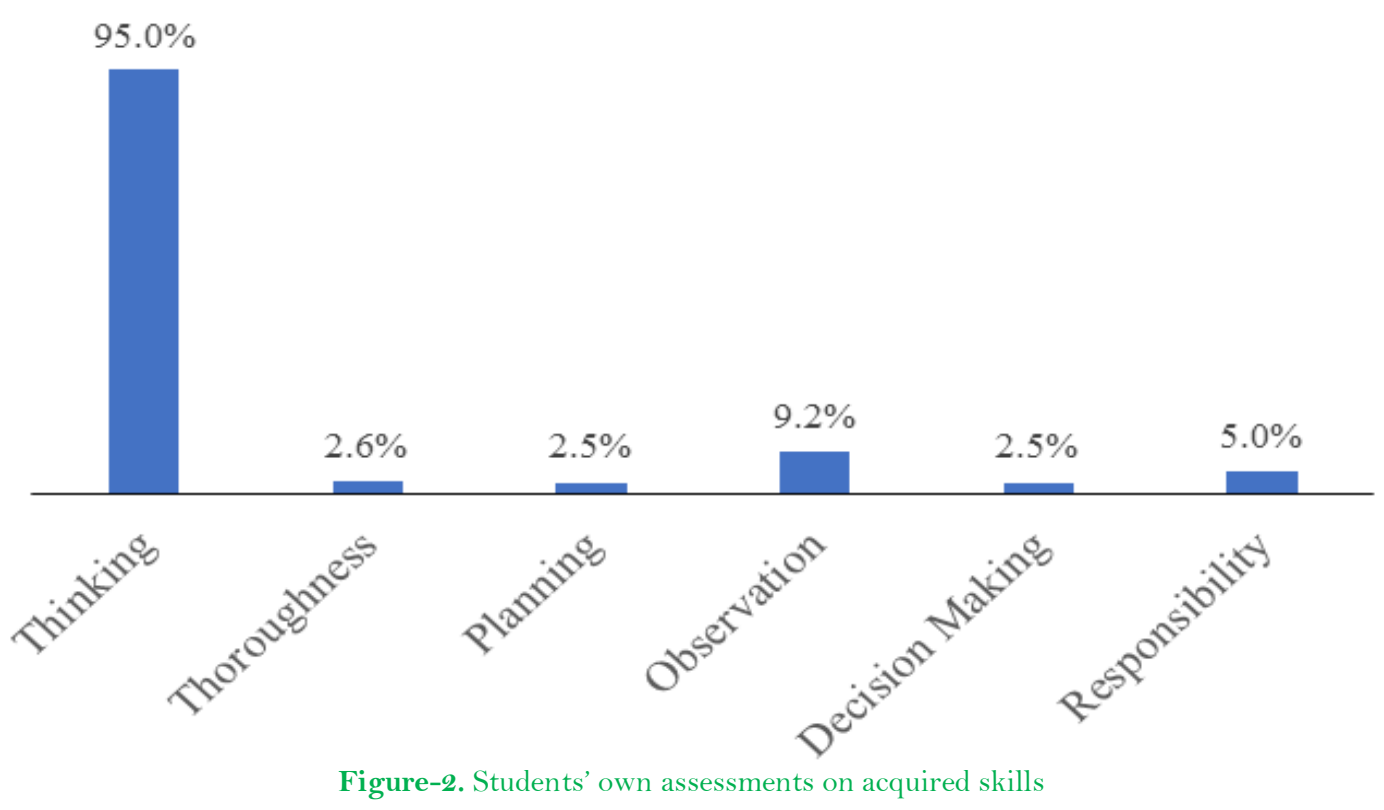

Students' general impressions of the lessons are exhibited in Figure 3. 72.5\% $(\mathrm{n}=29)$ saw the lessons as fun, challenging or exciting activities. They also expressed a wish for more lessons to be of active type (Student $3-I$ really liked this self-learning more than sitting in a seat. I wished other teahcers would do the same.) which had helped them stay focused (Student 20 - I got to plan my own work. I wanted to learn more.). This finding further confirms the conclusion in Teemuangsai and Meesook (2017) study which stated there is a need, in Thai classrooms, for change in the teaching style to make the lessons less boring, more engaging and relevant to real life. The students' reflections revealed instances where they viewed the lessons as enabling agents for self confidence $(n=1)$ and perseverance $(n=1)$. Also, $3 \%(n=1)$ reported a difficulty in group working environment.

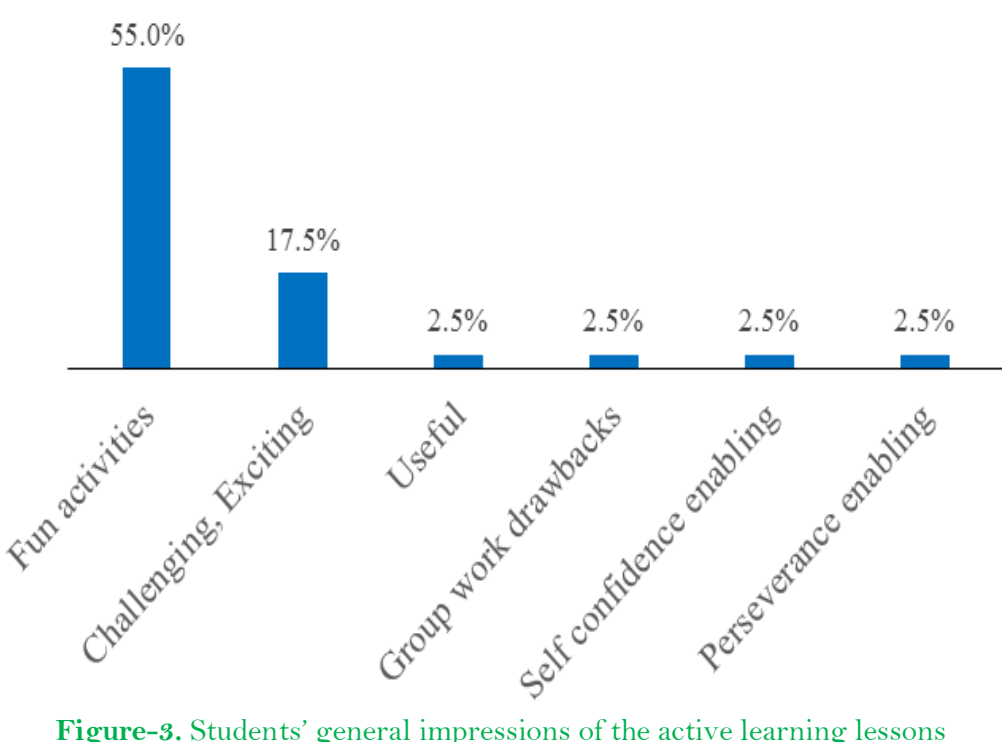

A follow up study after the study group has entered their teaching careers for 2 years displayed a strong influence of this active learning experience on their career practice. We were able to collect responses from 23 respondents which accounted for $57.5 \%$ of the subjects in this study. $91.3 \%(\mathrm{n}=21)$ of the respondents were classroom teachers and $8.7 \%(\mathrm{n}=2)$ worked in private sectors. Among classroom teachers, $85.7 \%(\mathrm{n}=18)$ clearly stated that credibility of information they acquired from online sources was their primary concern in lesson preparation, $57.1 \%(\mathrm{n}=12)$ applied their direct experience from this study in their teaching practice by creating platforms for their students to actively acquire information and filter credible contents. $38.1 \%(\mathrm{n}=8)$ of the respondents found the discussions within and among groups during the content analysis steps in this study helpful in conduction communications with colleagues in the workplace.

\section{Conclusion}

We believe that traditional passive learning is likely to limit the opportunity for any learners to independently develope and practice analytical thinking skills. By giving this platform of active learning and information credibility evaluation, the students were better prepared to be analytical-thinking teachers which in turn would be capable to produce analytical-thinking students. The follow-up study did provide a sign of hope for the latter as it showed some evidence of analytical thinking skill practice transferred from the subject group to their school students. Though the subject group represented only a small number of teacher students, the results of the analytical thinking skill fostering method in this study are encouraging. However, more analytical/critical thinking skill practice is needed in teacher students' preparation in order to further a possibility to shape up the critical thinking profile in Thailand's education. 


\section{References}

Bachhel, R. and R.G. Thaman, 2014. Effective use of pause procedure to enhance student engagement and learning. Journal of Clinical and Diagnostic Research, 8(8): XMo1-XM03.

Chowdhury, F., 2016. The power of using pause procedure during accounting lecture: An action research study. European Journal of Business and Social Sciences, 5(06): 101-108.

Climer, N., M. King, K. Miranda, I. Moon, B. Uapipatanakul and S. Zamora, 2009. Promoting active teaching methods in rural Thailand: A case study on science laboratory activities. Undergraduate Report, Worcester Polytechnic Institute.

Forehand, M., 2005. Bloom's taxonomy: Original and Revised. In M. Orey (Ed.), Emerging Perspectives on Learning. Teaching, and Technology (E-Book). pp: 41-47. Available at https:// textbookequity.org/Textbooks/Orey_Emergin_Perspectives_Learning.pdf.

Henschke, J.A. and A. Ratana-Ubol, 2015. Cultural learning processes through local wisdom: A case study on adult and lifelong learning in Thailand. International Journal of Adult Vocational Education and Technology, 6(2): 41-60. Available at: https://doi.org/10.4018/ijavet.2015040104.

Kim, S., 2010. Questioners' credibility judgments of answers in a social question and answer site. Information Research, $15(2): 5$.

Metzger, M.J. and A.J. Flanagin, 2013. Credibility and trust of information in online environments: The use of cognitive heuristics. Journal of Pragmatics, 59: 210-220. Available at: https://doi.org/10.1016/j.pragma.2013.07.012.

Metzger, M.J., A.J. Flanagin and R.B. Medders, 2010. Social and heuristic approaches to credibility evaluation online. Journal of Communication, 60(3): 413-439. Available at: https://doi.org/10.1111/j.1460-2466.2010.01488.x.

Mungmachon, M.R., 2012. Knowledge and local wisdom: Community treasure. International Journal of Humanities and Social Science, 2(13): $174-181$.

Office of the Education Council, 1999. National education act B.E.2542 and amendments (Second National Education Act B.E.2544 (2002). Bangkok: Office of the Educational Council.

Office of the National Education Commission, 1997. Constitution of the Kingdom of Thailand, B.E.2540. Bangkok: Printer Department of Religion.

Ratana-Ubol, A., 2016. Enhancing local wisdom through lifelong learning in Thailand. Culture, Biography \& Lifelong Learning, 2(2): 57-68.

Sopa, M., 2018. Local wisdom in the cultural symbol of Indonesian traditional house. The 1st Annual International Conference on Language and Literature, KnE Social Sciences. pp: 524-531.

Teemuangsai, S. and C. Meesook, 2017. Thailands classroom learning practices in secondary level: Are we ready for learning in the 21 stcentury? International Journal of Science and Technology Education Research, 8(1): 1-12. Available at: https://doi.org/10.5897/ijster20 17.0403. 